This item was submitted to Loughborough's Research Repository by the author.

Items in Figshare are protected by copyright, with all rights reserved, unless otherwise indicated.

Moisture absorption-desorption effects in adhesive joints

PLEASE CITE THE PUBLISHED VERSION

PUBLISHER

(c) Elsevier

VERSION

AM (Accepted Manuscript)

LICENCE

CC BY-NC-ND 4.0

REPOSITORY RECORD

Mubashar, Aamir, lan A. Ashcroft, Gary W. Critchlow, and A.D. Crocombe. 2009. "Moisture Absorptiondesorption Effects in Adhesive Joints". figshare. https://hdl.handle.net/2134/4636. 
This item was submitted to Loughborough's Institutional Repository (https://dspace.lboro.ac.uk/) by the author and is made available under the following Creative Commons Licence conditions.

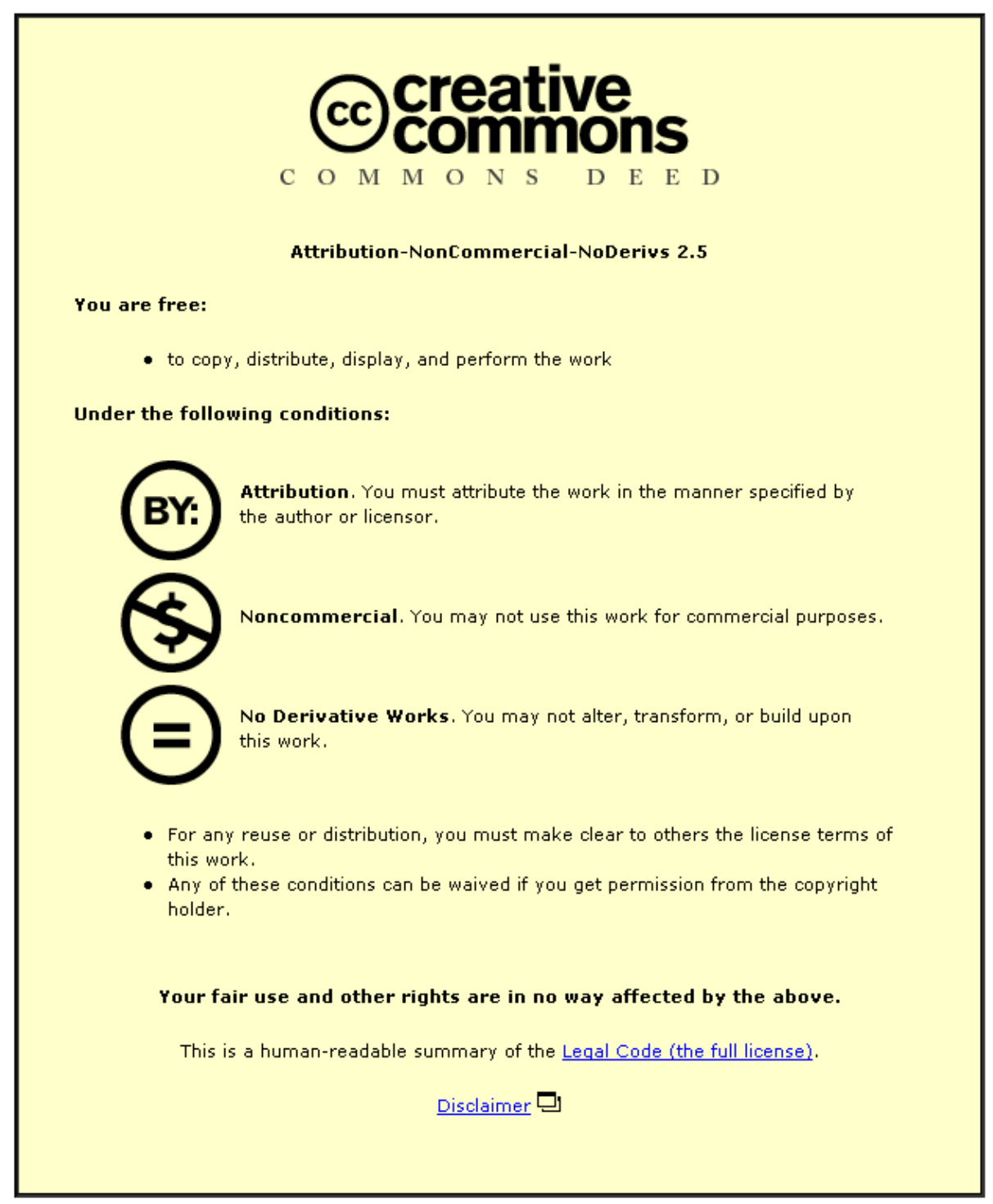

For the full text of this licence, please go to: http://creativecommons.org/licenses/by-nc-nd/2.5/ 


\title{
Moisture absorption-desorption effects in adhesive joints
}

\author{
A. Mubashar ${ }^{a}$, I. A. Ashcroft ${ }^{a, *}$, G. W. Critchlow ${ }^{b}$, A. D. Crocombe \\ ${ }^{a}$ Wolfson School of Mechanical and Manufacturing Engineering, Loughborough University, \\ Loughborough, Leicestershire LE11 3TU, UK \\ ${ }^{b}$ Institute of Surface Science \& Technology, IPTME, Loughborough University, Loughborough, \\ Leicestershire LE11 3TU, UK \\ ${ }^{c}$ Division of Mechanical, Medical, and Aerospace Engineering, University of Surrey, Guildford \\ GU2 $7 H X, U K$
}

\begin{abstract}
This paper presents a study of moisture absorption-desorption effects in single lap adhesive joints. Experiments were carried out to characterise the moisture uptake of the single part epoxide adhesive, FM73. Tensile testing of single lap joints manufactured from aluminium alloy $2024 \mathrm{~T} 3$ and $\mathrm{O}$ and FM73 adhesive was carried out after the joints were exposed to different conditioning environments. The experimental results revealed that the failure strength of the single lap joints with 2024 T3 adherends progressively degraded with time when conditioned at $50^{\circ} \mathrm{C}$, immersed in water. However, the joint strength almost completely recovered after moisture was desorbed. The single lap joints with $2024 \mathrm{O}$ adherends showed decreased strength for 28 days of conditioning, after which strength recovered, reaching a plateau after 56 days. Again, strength almost completely recovered on desorption of moisture. The strength recovery of the joints, after desorption of moisture, showed that the degradation of the adhesive was largely reversible. Analysis of the failure surfaces revealed that the dry joints failed cohesively in the adhesive layer and that the failure path moved towards the interface after conditioning. The failure mode then reverted back to cohesive failure after
\end{abstract}

\footnotetext{
* Corresponding author. Tel: +44 1509 223229; fax: +44 1509223934
} 
moisture desorption.

Keywords: Epoxy/Epoxides (A), Durability (P), Aluminium and alloys (S), Moisture Diffusion

\section{Introduction}

The degradation of adhesively bonded joints through the effects of moisture is one of the major concerns affecting their wide implementation in structure applications. Moisture ingress in an adhesive joint with non-absorbing adherends may occur through the bulk adhesive and in the interfacial region between adhesive and the adherends. The later is sometimes referred to as the "interphase". Moisture influences the adhesive by plasticisation and swelling [1], which may decrease joint strength [2]. In addition, water can disrupt interfacial bonds in a causing non-reversible damage, as predicted by the theories of adhesion, or cause failure by corrosion of the underlying metal substrate. Fickian diffusion has been used by researchers to predict moisture concentration in adhesives $[3,4]$. In Fickian diffusion it is assumed that the moisture flux is directly proportional to the concentration gradient in a material. The solution to the Fickian diffusion for a plane sheet of thickness $2 l$ was presented by Crank [5] and is given by:

$$
C_{t}=\left(1-\frac{4}{\pi} \sum_{n=0}^{\infty} \frac{(-1)^{n}}{2 n+1} e^{\frac{-D(2 n+1)^{2} \pi^{2} t}{4 l^{2}}} \cos \frac{(2 n+1) \pi x}{2 l}\right) \times C_{\infty}
$$

where $C_{t}$ is the moisture concentration at any time interval $t, C_{\infty}$ is the saturated moisture concentration, $D$ is the diffusion coefficient and $x$ is the spatial coordinate. The mass uptake $M_{t}$ at any time interval $t$, obtained by integrating Equation (1) over the domain, is given by: 


$$
M_{t}=\left(1-\frac{8}{\pi^{2}} \sum_{n=0}^{\infty} \frac{1}{(2 n+1)^{2}} e^{\frac{-D(2 n+1)^{2} \pi^{2} t}{4 l^{2}}}\right) \times M_{\infty}
$$

where $M_{\infty}$ is the saturated mass uptake. Fickian diffusion is observed in polymers well above their glass transition temperature $\left(\mathrm{T}_{\mathrm{g}}\right)$ [6]. At temperatures below $\mathrm{T}_{\mathrm{g}}$, a nonFickian moisture uptake is observed, where the diffusion process deviates from Fickian behaviour after initial uptake. Several models have been suggested to predict nonFickian uptake behaviour [7-10], including a dual Fickian model, which is based on two Fickian processes [11]. The two mechanisms of the dual Fickian model are considered to be working in parallel, where the concentration at any point may be determined by:

$$
\begin{aligned}
& C_{t}=\left(1-\frac{4}{\pi} \sum_{n=0}^{\infty} \frac{(-1)^{n}}{2 n+1} e^{\frac{-D_{1}(2 n+1)^{2} \pi^{2} t}{4 l^{2}}} \cos \frac{(2 n+1) \pi x}{2 l}\right) \times C_{1 \infty}+ \\
& \left(1-\frac{4}{\pi} \sum_{n=0}^{\infty} \frac{(-1)^{n}}{2 n+1} e^{\frac{-D_{2}(2 n+1)^{2} \pi^{2} t}{4 l^{2}}} \cos \frac{(2 n+1) \pi x}{2 l}\right) \times C_{2 \infty}
\end{aligned}
$$

where $C_{1 \infty}$ and $C_{2 \infty}$ are fractions of saturated concentration $C_{\infty}, D_{1}$ and $D_{2}$ are the diffusion coefficients and $l$ is the length of the diffusion path. The mass uptake for the dual Fickian model at any time $t$ is given by:

$$
\begin{aligned}
& M_{t}=\left(1-\frac{8}{\pi^{2}} \sum_{n=0}^{\infty} \frac{1}{(2 n+1)^{2}} e^{\frac{-D_{1}(2 n+1)^{2} \pi^{2} t}{4 l^{2}}}\right) \times M_{1 \infty}+ \\
& \left(1-\frac{8}{\pi^{2}} \sum_{n=0}^{\infty} \frac{1}{(2 n+1)^{2}} e^{\frac{-D_{2}(2 n+1)^{2} \pi^{2} t}{4 l^{2}}}\right) \times M_{2 \infty}
\end{aligned}
$$

where $M_{1 \infty}$ and $M_{2 \infty}$ are fractions of saturated mass uptake $M_{\infty}$. 
In the case of epoxy adhesives, it has been suggested that moisture absorption produces irreversible damage to the adhesive and that the strength of the adhesive is not fully recoverable after removal of moisture. For example, Apicella et al [12] proposed that the moisture diffusion caused irreversible damage by producing microcavities in the adhesive. Hand et al [2] also considered that microcavities were responsible for the loss of cohesive strength in inherently tough adhesives. Lin and Chen [13] suggested that hygrothermal ageing is irreversible and lowers the glass transition temperature $\left(\mathrm{T}_{\mathrm{g}}\right)$ of DGEBA/DDA adhesive systems by about $20^{\circ} \mathrm{C}$. It was concluded that the moisture absorption had permanently damaged the epoxy system. Bao et al [14] studied moisture absorption and hygrothermal aging in a bismaleimide resin. It was suggested that moisture caused plasticisation and swelling of the resin, resulting in a more open and accessible network structure. On desorption, the plasticisation was removed but it was shown that the structural relaxation was irreversible upon drying of water.

The decrease in mechanical properties of the adhesive on absorption of moisture is also accompanied by a loss of joint strength [15]. The joint strength does not decrease to zero but becomes constant at a certain level [16]. Minford [1] showed that the shear strength of aluminium / epoxy joints recovered when exposed to 5\% and $100 \% \mathrm{RH}$ and then dried at $90^{\circ} \mathrm{C}$. Similarly, Comyn and co-workers [17] conditioned aluminium joints bonded with modified epoxide adhesives at $50^{\circ} \mathrm{C}, 100 \%$ relative humidity $(\mathrm{RH})$ for 5000 hours and then partially dried them at 50\% RH for a further 5000 hours. Some strength recovery was observed after drying the joints, however, full strength recovery of joints was not seen in any case. The recovery of strength was attributed to the reversible effect of plasticisation while the irrecoverable strength was attributed to irreversible disruption at the interface due to the effect of moisture [16]. 
Many authors [18-20] have reported that the mechanics of failure changes from cohesive failure in the adhesive to apparent interfacial failure with increasing amount of moisture. Based on this, it was suggested by Kerr et al [21] and Butt and Cotter [22] that interfacial weakening was responsible for the decreased strength in conditioned joints.

Analysis of failed joint surfaces provides clues to the nature of failure and the underlying mechanisms. Various methods, including, optical microscopy, scanning electron microscopy (SEM), X-ray photoelectron spectroscopy (XPS) and infra-red spectroscopy (IR) have been employed for this purpose [23]. In well prepared epoxy / aluminium joints, failure is generally cohesive in dry joints. The failure path becomes more complex under wet conditions and a mixed mode failure can be observed. It has been observed that the failure path in cyclic fatigue tested joints under wet conditions is mainly through the aluminium-oxide layer or at the primer / adhesive / aluminiumoxide interphase $[24,25]$. The surfaces of the aluminium in this work were prepared by chromic acid etching (CAE) or grit blasting after degreasing. Both of these treatments produce inferior surfaces to the anodising procedure used in the present study.

The study of moisture absorption-desorption characteristics of structural adhesives provides a basis for the predictive modelling of adhesive joints. Predictive modelling can reduce uncertainties in the use of adhesive joints by providing information about the residual strength of joints after extended periods in service. The present work explores the change and recovery of strength in toughened epoxy / aluminium single lap joints. The joints were conditioned at $50^{\circ} \mathrm{C}$, immersed in water for a maximum duration of 182 days. During this period, sets of joints were removed at predetermined intervals and tensile tested to determine the failure strength. Duplicate sets of the conditioned 
joints were dried to remove moisture and the degree of strength recovery was determined by tensile testing. The failure surfaces of these joints were examined using digital image analysis, SEM and XPS. This investigation helps to explain the mechanisms of strength reduction and stress recovery observed during the experimental work. Finite element modelling was performed to predict the moisture concentration in the adhesive layer during the conditioning.

\section{Experimental}

\subsection{Characterisation of Moisture Diffusion}

The structural adhesive FM73 manufactured by Cytec Engineered Materials was used in this study. FM73 is a rubber toughened epoxy that comes with a polyester knit carrier for support and handling purposes. The manufacturer's recommended curing cycle for FM73 includes heating to $120^{\circ} \mathrm{C}$ for one hour [26] and the $\mathrm{T}_{\mathrm{g}}$ of the adhesive has been reported to be 98.12 to $99.7^{\circ} \mathrm{C}[27,28]$.

Bulk samples of $1 \mathrm{~mm}$ thickness were prepared by stacking multiple layers of the adhesive film, each of $0.12 \mathrm{~mm}$ thickness. During manufacture, the layers were compressed using a steel roller to ensure that air trapped between the layers was released as this helps to minimise the formation of voids during curing. The adhesive layup was placed in a mould and cured in a hot press at $120^{\circ} \mathrm{C}$ for one hour [26]. The bulk samples, of dimensions $60 \times 40 \times 1 \mathrm{~mm}$, were cut from the cured panel.

The moisture diffusion properties of the adhesive were determined by the gravimetric method. Before commencing the environmental conditioning, the bulk samples were dried in an oven at $50^{\circ} \mathrm{C}$ to constant weight. The samples were then subjected to a single absorption-desorption cycle in water at $50^{\circ} \mathrm{C}$. A Mettler Toledo AL204 electronic 
balance with $0.1 \mathrm{mg}$ accuracy was used to weigh the samples at predetermined time intervals during conditioning.

\subsection{Preparation and Testing of Single Lap Joints}

Single lap joints were used to determine changes in joint strength after moisture absorption. The adherends were prepared from aluminium 2024 alloy (A12024). The A12024 is available in unclad and clad forms, where in the clad form the surface is coated with a layer of pure aluminium. In this work, unclad A12024 was used in both a solution heat treated (T3) and soft state (O), in order to investigate the effects of adherend strength and yielding on joint strength. The environmental conditioning of the joints was carried out at $50^{\circ} \mathrm{C}$, immersed in deionised water.

The single lap joints were prepared according to BS ISO 4587:2003 [29] and their configuration and geometry is shown in Figure 1. The adherends were cut from $3.2 \mathrm{~mm}$ thick A12024 T3 and O sheets. The surfaces of the adherends were prepared by degreasing followed by ACDC anodising. ACDC anodising is an environmentally friendly process, which is free from the hexavalent chromium found in the aerospace industry standard Chromic Acid Anodising (CAA).

During ACDC anodising, the adherends alternatively act as anodes and cathodes. The adherends were suspended in an overall 5\% solution of phosphoric and sulphuric acid. An alternating current (AC) was applied at a voltage of $15 \mathrm{~V}$ for 2 minutes at $35^{\circ} \mathrm{C}$. This process was followed by the application of direct current (DC) with a voltage of $20 \mathrm{~V}$ for 10 minutes at $35^{\circ} \mathrm{C}$. The ACDC anodising process provides a high energy, porous oxide surface with a dense, corrosion resistant layer adjacent to the aluminium. The surface was rinsed with water after anodising and adherends were dried in air. Further details of the ACDC pretreatment may be found in [30]. The ACDC pretreatment was 
followed by the application of BR127 corrosion inhibiting primer. The primer was air dried for 30 minutes and then cured for one hour at $120^{\circ} \mathrm{C}$.

The adhesive was brought to room temperature in a desiccator from its storage temperature of $-24^{\circ} \mathrm{C}$ before bonding and the bonded adherends were held together by clips. The bondline thickness was maintained by the carrier knit in the adhesive film. The adhesive was cured at $120^{\circ} \mathrm{C}$ for one hour, giving a bondline thickness of $0.12 \pm 0.02 \mathrm{~mm}$. Small fillets were formed at the edges of the overlap due to overflow of the adhesive.

A set of three joints was tensile tested at room temperature using a Hounsfield H20K-W tensometer to obtain the failure strength of unconditioned joints. The rest of the joints were conditioned in water at $50^{\circ} \mathrm{C}$. A set of six joints were removed from the conditioning environment at predefined intervals of 7, 14, 28, 56 and 182 days. Three joints from the extracted set were immediately tensile tested while the remaining three joints were placed in an oven for drying at $50^{\circ} \mathrm{C}$. Drying continued until complete desorption of moisture. After drying, the joints were brought to room temperature in a desiccator and tensile testing was carried out. The joints dried after conditioning will hereafter be referred to as dried joints.

\subsection{Failure surface characterisation}

The exposed surfaces from tested joints were analysed to determine the failure characteristics and locus of failure. Two types of failure could be identified by image analysis. The first was failure within the adhesive layer, which will be referred to as "cohesive" failure. The second appeared to at the interface between the adhesive and aluminium adherend and this will be referred to as "apparent interfacial failure". Digital image analysis was used as a first step to quantify the proportions of cohesive and 
apparent interfacial failure. The failure surface was digitised using a Reichert MEF-3 Microscope with a macro arm attachment and the image was converted to a grey scale image to obtain high contrast between different image areas. The grey scale image was further converted to a two bit image using the mathematical software Matlab. A Matlab subroutine was then used to determine the percentage area covered by adhesive and "metal". The same procedure was carried out on both adherends of a failed joint.

The surfaces of adherends were also examined using a LEO 1530 VP ultra-highresolution scanning electron microscope (SEM) operating with a primary electron beam of $5 \mathrm{kV}$ and a current of approximately $200 \mathrm{pA}$. To determine the locus of failure, the failure surfaces were further analysed using X-ray Photoelectron Spectroscopy (XPS) where an ESCALAB MK II surface analysis system using an $\mathrm{Al} \mathrm{K \alpha} \mathrm{X}$-ray source operating at a power of $160 \mathrm{~W}$ was used. The interface between adhesive and adherend is composed of several layers of oxide, primer and adhesive. XPS provides material composition from a depth of a few nanometres (about 10nm) of the exposed surface and thus has been successfully used in the past for the analysis of fracture surfaces in adhesive joints [24, 25, 31]. XPS data was obtained from an area of approximately 2 mm diameter, full width, half maximum (FWHM). Quantification was achieved using theoretically derived relative sensitivity factors based upon ionisation cross-sections and known instrument parameters and verified using standards of known composition. Peak areas were measured following the removal of a shirley-type background.

\section{Modelling Moisture Diffusion in the Adhesive}

Figure 2 shows the normalised mass uptake as a function of $\sqrt{t} / l$ from the absorption and desorption experiments. Repeatability of the tests was good with a standard deviation of $11 \%$. Considering the moisture uptake, it can be seen that the rate of 
absorption is faster during the initial stages of diffusion. At later stages, the absorption deviates from the initial uptake trend and follows a different gradient. The overall diffusion coefficient does not remain constant and changes with increasing concentration. After reaching an apparent equilibrium, the absorption plot shows a sudden increase in mass uptake at $\sqrt{t} / \mathrm{l}$ of approximately $28 \sqrt{\mathrm{hr}} / \mathrm{mm}$. Similar behaviour has also been reported previously, for a DGEBA/dicy based adhesive [32]. It is clear from the experimental observations that Fickian diffusion based on a single absorption process is inadequate in explaining the moisture uptake.

In the case of desorption, diffusion takes place in a single step until a constant weight is achieved as in a Fickian diffusion process. It was noted that the bulk adhesive samples achieved their original weight after desorption. The desorption process was faster than the absorption, which is an indication of changing adhesive structure due to moisture ingress.

Full saturation was not achieved during absorptions as the samples were conditioned for a predetermined time. $\mathrm{M}_{\infty}$ and $D$ were estimated by least square fitting of analytical models to the experimental data. The curve fitting was carried out in MathCAD using the genfit function, which employs an optimised Levenberg-Marquardt method [33]. Three different analytical models were used to determine the best curve fit to the experimental data and the resulting plots are shown in Figure 2a. The Fickian and dual Fickian models were not able to adequately represent the moisture uptake owing to the presence of the secondary uptake in the experimental data. To incorporate the secondary uptake in the analytical model, a dual Fickian model with a Heaviside step function was used. A Heaviside step function returns zero for negative values and one for positive values. This model is termed the "delayed" dual Fickian model, where the 
secondary uptake is modelled by power law. The mass uptake by a delayed dual Fickian model is, hence, given by:

$$
\begin{aligned}
& M_{t}=\left(1-\frac{8}{\pi^{2}} \sum_{n=0}^{\infty} \frac{1}{(2 n+1)^{2}} e^{\frac{-D_{1}(2 n+1)^{2} \pi^{2} t}{4 l^{2}}}\right) \times M_{1 \infty}+ \\
& \left(1-\frac{8}{\pi^{2}} \sum_{n=0}^{\infty} \frac{1}{(2 n+1)^{2}} e^{\frac{-D_{2}(2 n+1)^{2} \pi^{2} t}{4 l^{2}}}\right) \times M_{2 \infty}+\Phi\left(t-t_{1}\right)\left(a t^{b}+c\right)
\end{aligned}
$$

where $\Phi$ is the Heaviside step function, $t_{1}$ is the start time of secondary uptake as determined experimentally and $a, b$ and $c$ are the power law constants determined by curve fitting. The desorption process was modelled using Fickian diffusion and it is seen in Figure $2 \mathrm{~b}$ that this provides a good fit to the desorption plots.

The spatial distribution of moisture concentration in the adhesive layer of the simple lap joints was determined using the finite element method. The commercially available finite element code, Abaqus, was used, where only a quarter of the single lap joint was modelled due to geometric and loading symmetry, as shown in Figure 3a. Fillets were included at the end of the adhesive layer in the models based on average dimensions measured from manufactured joints. Three dimensional continuum field elements were used, with a minimum element size of $0.12 \times 0.06 \times 0.12 \mathrm{~mm}$. The mesh of the adhesive layer is shown in Figure $3 \mathrm{~b}$. As the adherends were prepared by degreasing and the ACDC pretreatment (Section 2.2), which promoted good wetting of the adherends, the potential of enhanced moisture diffusion along the interface is reduced and, hence the main moisture path was considered to be through the adhesive. Normalised moisture concentration was applied as a boundary condition and a transient solution was obtained for moisture absorption at 7, 14, 28, 56 and 182 days. The dual Fickian model was implemented by running two sequential analyses with $D=D_{1}$ and $D=D_{2}$ and a script 
was used to add the concentration at each integration point, providing the dual Fickian moisture uptake. The moisture diffusion step was followed by the application of a load of $8 \mathrm{kN}$ to determine the stresses in the joint after various levels of moisture diffusion.

\section{Results}

Figure 4 plots the failure load as a function of conditioning time for the single lap joints. It can be seen that the strength of unconditioned A12024 O joints is significantly less than that of A12024 T3 joints. This is because of the plastic deformation of the A12024 O adherends during tensile testing, which occurs because of the lower yield stress of A12024 O (75.8 MPa) compared to A12024 T3 (345 MPa).

The A12024 T3 joints exhibited a progressive decrease in strength with conditioning time. When A12024 T3 joints were dried after conditioning for 7 days and tensile tested, they achieved at least $98 \%$ of the original strength. The recovered percentage of the original strength is similar for all moisture absorption intervals. The A12024 O joints showed a more rapid decrease in strength than the A12024 T3 joints for the initial conditioning period of 14 days. However, a strength increase was then observed after tensile testing for 28 days of conditioning. After moisture absorption for 56 days, the strength of the joint recovered to about $86 \%$ of its original strength and remained at a similar value after conditioning of 182 days. The A12024 O joints were also tested after drying and the strength recovered to $88 \%$ or more of the unconditioned joint strength.

The two main types of failure observed in the joints were cohesive failure and apparent interfacial failure. Cohesive failure in the adhesive layer was observed in the single lap joints tested in the unconditioned state. Representative failure surfaces for cohesively failed A12024 T3 and A12024 O joints are shown in Figures 7a and b respectively. With 
the conditioned A12024 T3 joints, cohesive failure predominated in joints tested after 7 , 14, 28 and 56 days, whereas, a mixed failure was observed in joints conditioned for 182 days. However, patches of apparent interfacial failure were present to some degree on most of the failure surfaces. A similar pattern of cohesive or mixed failure was observed in the dried joints. A quantitative assessment of the failure surfaces was carried out by digital image analysis and is discussed in later sections.

Considering the A12024 O joints, failure in unconditioned joints occurred in the adhesive layer as seen in Figure 5b. After conditioning for 7 days, a mixed failure comprised of cohesive and apparent interfacial areas was observed. The failure started as apparent interfacial failure at the edges of the overlap and progressed to cohesive failure towards the middle of the overlap. Mixed failure was also observed when joints were tested after 14 days and 28 days of environmental conditioning, as seen in Figure $6 \mathrm{~b}$ and c. The dried A12024 O joints also showed a mixed failure, as shown in Figure $6 \mathrm{f}$ and g, for 7 and 14 days respectively. However, cohesive failure was observed after 28 days, as seen in Figure $6 \mathrm{~h}$.

Figure 7 shows the predicted normalised moisture concentration in the adhesive after different conditioning intervals. A quarter portion of the adhesive layer is shown where moisture starts to ingress from the exposed surfaces of the adhesive and moves towards the middle. After 7 days of moisture absorption, moisture has reached significant levels in the fillets and edges of the adhesive layer, however, most of the adhesive layer remains dry. With increasing conditioning time, the moisture front keeps progressing towards the middle of the adhesive layer. Figure 8 plots the normalised moisture concentration in the middle of the adhesive layer at different conditioning times. It can 
be seen that full saturation of the adhesive layer was not achieved even after the full 182 days of exposure.

\section{Analysis of Failure Surfaces}

\subsection{Failure Type}

The fracture surfaces were analysed by digital image analysis, as discussed in Section 2.3. The amount of adhesive on the adherends was used in the quantification and a cohesive failure was represented by unity. Mixed failure was thus quantified as a fraction of the amount of adhesive left on the adherend to the amount of adhesive on a cohesively failed surface. Figure 9 shows a comparison of failure load and fractional adhesive area at different moisture absorption intervals for $\mathrm{T} 3$ joints. The fraction of cohesive failure decreases i.e. the amount of apparent interfacial failure increases with the decrease in failure load at 7 and 14 days of moisture absorption. However, the fraction of cohesive failure increases after 28 and 56 days of moisture absorption, finally decreasing to a minimum of $59 \%$ after 182 days of moisture absorption.

The analysis of dried A12024 T3 joints also showed mixed failure at all conditioning intervals. The digital image analysis results are shown in Figure 10. The fraction of adhesive area i.e. cohesive failure decreased after 7 days of moisture absorption. Then an increase in fractional cohesive failure was seen for 14 and 28 days of moisture absorption and finally the fraction of cohesive failure decreased again after 56 days of moisture absorption. Although the fraction of cohesive failure area does not exactly follow the trend of failure load, the failure remained mainly cohesive. The mixed cohesive and apparent interfacial failure of joints means that the fracture path travels both through the adhesive as well as within the interfacial regions. This may be 
attributed to the degradation in the adhesive and primer properties. As already mentioned, plasticisation of the adhesive caused by moisture ingress causes degradation of the elastic modulus and lower failure strength would be expected, as observed.

A comparison of failure load and failure type for conditioned A12024 O joints is shown in Figure 11. The proportion of cohesive failure within the adhesive shows a good correlation with failure load. As the failure load of the joint decreases after 7 and 14 days of moisture absorption, the failure surface shows more apparent interfacial failure. The strength of the joint started recovering after 28 days of moisture absorption and recovery continued until the end of testing. This coincided with further surfaces with less apparent interfacial failure. Interestingly, similar trends were seen in the failure surfaces of joints which were tested after drying, as can be seen Figure 12.

Consideration of Figures 12 to 15 indicates that there is a commonality between joint strength and failure type, with lower joint strength corresponding to increased apparent interfacial failure. Another common trend in these figures is a decrease and recovery in proportion of cohesive failure between zero and 56 days exposure. This is more pronounced in the joints with A12024 O adherends and corresponds to a similar drop and recovery in joint strength.

\subsection{XPS Studies}

A complex interphase exists between the aluminium adherends and the bulk adhesive layer. The oxide layer developed on the adherends has a highly porous structure, as illustrated in Figure 13. This enables penetration by the primer which provides strong mechanical interlocking. The primer was applied to improve the bonding and corrosion characteristics of the surface. The apparent interfacial failure may be originating in any of the interface layers and may travel through multiple layers. 
XPS analysis was carried out to investigate the locus of failure where apparent interfacial failure was observed. As mentioned earlier, the failure of unconditioned joints was predominately cohesive failure of the adhesive layer. The results of the XPS analysis of the A12024 O and $\mathrm{T} 3$ joints are shown in Table 2. Beginning with the A12024 T3 joints, the unconditioned joints were initially analysed in order to establish a baseline, indicative of cohesive failure. In Table 2, the adherends of a failed joint are referred to as "metal" and "adhesive" side irrespective of the type of failure. For the unconditioned A12024 T3 joint, both failure surfaces showed large amounts of carbon. Nitrogen was also detected, which is indicative of failure in the adhesive layer. A small amount of aluminium was detected on the metal side, which may be the result of signal detection from the edge of the sample or due to surface contamination during the sample preparation process. Aluminium was not detected on the adhesive side.

A12024 T3 joints conditioned for 56 days were also analysed with XPS. As seen in Figure 10, these joints have more apparent interfacial failure than the unconditioned joints. A small amount of aluminium was detected on the adhesive side, which was similar to the earlier observation of aluminium in the cohesive failure and may not be an indication of oxide layer failure. A failure through the oxide layer would have shown a large amount of aluminium on both the metal and adhesive sides. The metal side also had only a small amount of aluminium present. The amount of carbon on both the metal and adhesive sides was high, indicating failure was either through the adhesive layer or through the interphasial polymer. Nitrogen was also detected on both sides of the failure surface. This suggests that even though some patches of apparent interfacial failure were present; the failure predominately occurred within or between the adhesive and primer layer, remaining largely cohesive. 
In the case of the A12024 O joints, the surfaces of unconditioned joints with predominately cohesive failure had similar composition to the A12024 T3 joints. The adhesive side had no aluminium while a very small amount of aluminium was detected on the metal side. The large amount of carbon and presence of nitrogen, as found in a cohesive failure, were also present.

The Al2024 O joints conditioned for 7, 14 and 28 days exhibited large areas of apparent interfacial failure, as indicated in Figure 6a-c. The maximum reduction in joint strength was observed after 14 days of conditioning, with $46 \%$ of apparent interfacial area. When analysed using XPS, it was seen that a small amount of aluminium was present on the adhesive face of an A12024 O joint conditioned for 14 days. The carbon percentage was about $80 \%$, the source of this is most likely to be the epoxy or primer. The metal face of the joint, however, showed a significantly higher concentration of the aluminium but also showed presence of nitrogen on the surface. The nitrogen indicates that there might be a very thin layer of primer present on the metal surface. The percentage of carbon on the metal side is also slightly higher than the expected percentage resulting from an oxide failure surface. Another reason for the high amount of aluminium on the metal side may be that the analysis depth of XPS exceeds the thickness of a very thin residual primer layer left on the adherend. The presence of primer was further investigated by scanning electron microscopy and is discussed later.

The highest amount of interfacial failure in dried A12024 O joints was observed after conditioning for 7 days and then drying. XPS analysis revealed that the adhesive side did not have any aluminium and a high percentage of carbon was present. The metal side had a large percentage of aluminium, indicating that the failure had occurred close 
to the oxide layer. The failure surface composition was similar to the apparent interfacial failure surfaces observed in conditioned A12024 O joints, discussed earlier.

As mentioned above, the XPS analysis of the surfaces, which had undergone apparent interfacial failure, pointed towards the possibility that a very thin layer of primer may be present on the metal side of failed joints and failure had not been through the oxide layer. This was further investigated by performing scanning electron microscopy. This allowed for high magnification (x50000) images of the apparent interfacial area. Upon close inspection, tiny particles were observed spread throughout the failure surface. These particles may serve as a fingerprint to identify the presence of the thin layer of primer.

A comparative failure surface of the primer was obtained by preparing a joint with the same surface preparation as the conditioned joints i.e. degreasing followed by ACDC anodising. However, the adherends were joined together using only primer BR127, i.e. without adhesive. The joint was subjected to tensile testing and the failure surfaces were observed using SEM, as shown in Figure 14. Primer BR127 is an epoxy phenolic system, which contains strontium chromate for corrosion inhibition [34]. This was observed in the form of tiny particles in the failed primer joint. Similar particles were also observed in the failure surfaces of conditioned joints. By comparison of the two surfaces and using these primer particles as a fingerprint, it may be deduced that a thin layer of primer may be present even in the case of an apparent interfacial failure. It is likely, therefore, that the failure occurred in the primer layer close to the oxide layer but did not travel into the oxide layer. 


\section{Discussion}

The degradation of joint strength may be due to deterioration of the adhesive system i.e. FM73 and BR127, the interface or a combination of the two. Moisture affects the adhesive in the form of plasticisation and swelling. A combination of both chemical and mechanical adhesion mechanisms may explain the nature of adhesion in the aluminium/epoxy joint system used in this work. The chemical theories attribute adhesion to polar and dispersive Van der Walls forces, acid base interactions, chemical bonds or metal organic complex formation [35-37]. The ACDC pretreatment, which was used in the present work, develops an oxide layer with a needle like structure at the interface of primer and the metal organic complex is also thought to be giving a "fibre reinforced" interphase. The mechanical interlocking theory is based on a lock and key effect achieved through the surface porosity of the adherends.

In the single lap joints, the interface between the primer and the oxide layer forms a non-regular path as the primer penetrates the oxide layer. Also, there is no distinct interface between the primer and the adhesive but an interphase is present. This is because primer BR127 is an epoxy phenolic and forms bonds with the epoxy adhesive FM73. In the case of a cohesive failure, the crack travels through the adhesive/primer layer and the failure may be attributed to the breakage of primary bonds. This suggests that the interface has strong adhesion forces in an unconditioned joint. In the case of conditioned joints, the adhesive and interface or interphase may be affected by the moisture uptake. The adhesive plasticises and swells, which decreases strength and the oxide layer may become hydrated. When cohesive failure was observed, it is suggested that the degradation of the interface is not considerable whilst the adhesive has degraded and caused the failure. 
In the case of apparent interfacial failure, it is important to consider that the primer and oxide layer are not only joined by interatomic forces, but a strong mechanical interlocking is also present, which would make complete separation of the primer from the oxide layer very difficult. Thus where apparent interfacial failure was observed, the SEM based analysis has shown that failure is within the primer layer, very close to the oxide layer. This allows for the explanation of strength recovery in cases when moisture was dried out of the joint. If it is considered that the diffused water caused disruption of inter-atomic forces at the interface, the re-establishment of strong secondary forces is difficult as they require very close contact between of the primer and adherend surfaces. This was possible during joint manufacture because of the flow of the primer. However, if the adhesive or primer layer fails cohesively, as is the case in this study, the removal of moisture by drying would recover the strength of the adhesive and primer and recovery of joint strength would be observed.

Recovery of strength was observed in A12024 O joints after conditioning for greater than 56 days, while no such phenomenon was observed in A12024 T3 joints. The only differentiating characteristic of the two types of joints is the strength of the adherends. When tested in the unconditioned state, the A12024 O joints undergo large deformations in the form of rotations and adherend yielding occurs before failure. After moisture absorption for 7 and 14 days, most of the adhesive layer still has low moisture concentration, as shown in Figures $10 \mathrm{a}$ and $\mathrm{b}$, but the adhesive may have become significantly weaker in the critical fracture initiation sites. Hence a combination of yielding in the adherends and a decrease in the strength of the adhesive and the primer in the failure initiation region may be responsible for the observed decrease in failure strength. After 56 or 182 days of moisture absorption, larger areas of the adhesive become plasticised (Figure $7 \mathrm{c}, \mathrm{d}, \mathrm{e}$ ), which results in reduced yielding and rotation of 
the adherends whilst there is little change in the moisture content and hence strength of the adhesive and primer in the areas of failure initiation. Figure 15a shows the change in failure strength and moisture concentration in the single lap joint with conditioning time when observed at a point in the fillet where failure initiates as shown in Figure 15b.

It can be seen that moisture concentration in the failure initiation area reaches almost complete saturation in 56 days and that this corresponds to a fall in adhesive strength in this area. The rotation and yielding of adherends occurred during tensile testing. Hence the combination of high interfacial strains through yielding and rotation of the adherends and the weakness of the adhesive in the area of failure initiation results in a decrease in strength, that recovers when plasticisation of the adhesive layer reduce the yielding and hence interfacial strains. As expected, this effect is much reduced when a high yield adherend is used.

\section{Conclusions}

Joint strength degradation followed by strength recovery after long moisture exposure times and subsequent dehydration of joints was observed during the conditioning and testing of single lap joints. Mixed cohesive and apparent interfacial failure modes were observed in the joints after conditioning. The loss of cohesive strength of the joints may be explained by the plasticisation of the adhesive or primer due to moisture absorption. In the case of apparent interfacial failure, surface analysis revealed that complete removal of the primer and adhesive from the surface of the adherend is not likely. This may be attributed to the porous nature of the oxide surface developed by the anodising process. Thus the presence of a very thin layer of primer was detected in cases of apparent interfacial failure. 
The recovery of joint strength; up to $100 \%$ of the original strength for A12024 T3 and $88 \%$ of the original strength for A12024 O was observed upon drying of conditioned joints. The strength recovery may be due to the reestablishment of the interatomic forces, however, primary bonds require a large amount of energy and it is unlikely that they will reform upon drying. Secondary bonding forces, such as London dispersion forces, may re-establish once the moisture is removed from the joint, however, the most significant influence of drying would be to recover the cohesive strength of the primer and adhesive. 


\section{References}

[1] Minford, J.D., Handbook of Aluminum Bonding Technology and Data. Marcel Dekker Inc, 1993.

[2] Hand, H.M., Arah, C.O., McNamara, D.K., Mecklenburg, M.F., Int. J. Adhesion and Adhesives. 1991;11:15-23.

[3] Wahab, M.A., Ashcroft, I.A., Crocombe, A.D., Shaw, S.J., The Journal of Adhesion. 2001;77:43-80.

[4] Brewis, D.M., Comyn, J., Tredwell, S.T., Int. J. Adhesion and Adhesives. 1987;7:3032.

[5] Crank, J., The Mathematics of Diffusion. Clarendon Press, 1975.

[6] Masaro, L.,Zhu, X.X., Prog. Polym. Sci. 1999;24:731-775.

[7] Wilde, W.P.D.,Shopov, P.J., Composite Structures. 1994;27:243-252.

[8] Dewimille, B.,Bunsell, A.R., J. Phys. D: Appl. Phys. 1982;15:2079-2091.

[9] Popineau, S., Rondeau-Mouro, C., Sulpice-Gaillet, C., Shahnahan, M.E.R., Polymer. 2005;46:10733-10740.

[10] Roy, S., Xu, W.X., Park, S.J., Liechti, K.M., J Appl Mech. 2000;67:391-396.

[11] Loh, W.K., Crocombe, A.D., Wahab, M.A., Ashcroft, I.A., Int. J. Adhesion and Adhesives. 2005;25:1-12.

[12] Apicella, A., Nicolais, L., Astarita, G., Drioli, E., Polymer. 1979;20:1143-1148.

[13] Lin, Y.C.,Chen, X., Polymer. 2005;46:11994-12003.

[14] Bao, L.-R., Yee, A.F., Lee, C.Y.C., Polymer. 2001;42:7327-7333.

[15] Comrie, R., Affrossman, S., Hayward, D., Pethrick, R.A., Zhou, X., Thompson, G.E., J. Adhesion. 2005;81:1199-1215.

[16] Bowditch, M.R., Int. J. Adhesion and Adhesives. 1996;16:73-79.

[17] Comyn, J., Brewis, D.M., Tredwell, S.T., J. Adhesion. 1987;21:59-78.

[18] Al-Harthi, M., Kahraman, R., Yilbas, B., Sunar, M., Aleem, A.B.J., Journal of Adhesion Science and Technology. 2004;18:1699-1710.

[19] Comrie, R., Affrossman, S., Hayward, D., Pethrick, R.A., Zhou, X., Thompson, G.E., Journal of Adhesion Science and Technology. 2006;20:1847-1872.

[20] Loh, W.K., Crocombe, A.D., Wahab, M.A., Watts, F.J., Ashcroft, I.A., Journal of Adhesion Science and Technology. 2002;16:1407-1429.

[21] Kerr, C., Macdonald, N.C., Orman, S., J. Appl. Chem. 1967;17:62-65. 
[22] Butt, R.I.,Cotter, J.L., J. Adhesion. 1976;8:11-19.

[23] Brewis, D.M.,Critchlow, G.W., J. Adhesion. 1995;54:175-199.

[24] Dickie, R.A., Haack, L.P., Jethwa, J.K., Kinloch, A.J., Watts, F.J., J. Adhesion. 1998;66:1-37.

[25] Abel, M.L., Adams, A.N.N., Kinloch, A.J., Shaw, S.J., Watts, F.J., Int. J. Adhesion and Adhesives. 2006;26:50-61.

[26] Cytec Engineered Materials, Datasheet - FM73 Toughened Epoxy Film. 1998.

[27] Baker, A., Rose, F., Jones, R., editors. Advances in the bonded composite repair of metallic aircraft structure. First ed. Vol. 1. 2002, Elsevier Science Ltd.

[28] Hojjati, M., Johnston, A., Hoa, S.V., Denault, J., J Appl Polym Sci. 2004;91:25482557.

[29] BS ISO 4587:2003 Adhesives - Determination of tensile lap-shear strength of rigidto-rigid bonded assemblies, British Standards Institution.

[30] Critchlow, G.W., Ashcroft, I.A., Cartwright, T., Bahrani, D., Anodising aluminium alloy, Patent No 2006, United Kingdom.

[31] Brewis, D.M.,Critchlow, G.W., Transactions of the Institute of Metal Finishing. 1976;74:198-201.

[32] De Neve, B.,Shanahan, M.E.R., Int. J. Adhesion and Adhesives. 1992;12:191-196.

[33] Mathcad 14 Help. 2007, Parametric Technology Corporation.

[34] Bishopp, J., Primers for adhesive bonding, in Handbook of adhesion, D.E. Packham, Editor. 2005, John Wiley \& Sons Ltd. p. 389-393.

[35] Aufray, M.,Roche, A.A., Int. J. Adhesion and Adhesives. 2007;27:387-393.

[36] Barthes-Labrousse, M.G., J. Adhesion. 1996;57:65-75.

[37] Kobozeva, I.A.,Petrova, I.A., Zhurnal Prikladnoi Spektroskopii. 1990;52:607-611. 


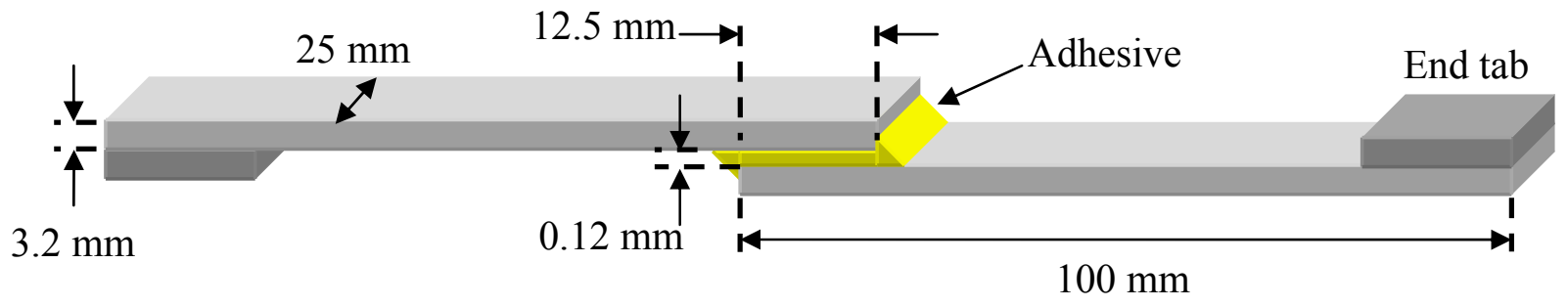

Figure 1: Geometry of Single lap joint with Al 2024 adherends and FM73 epoxy adhesive (not to scale) 


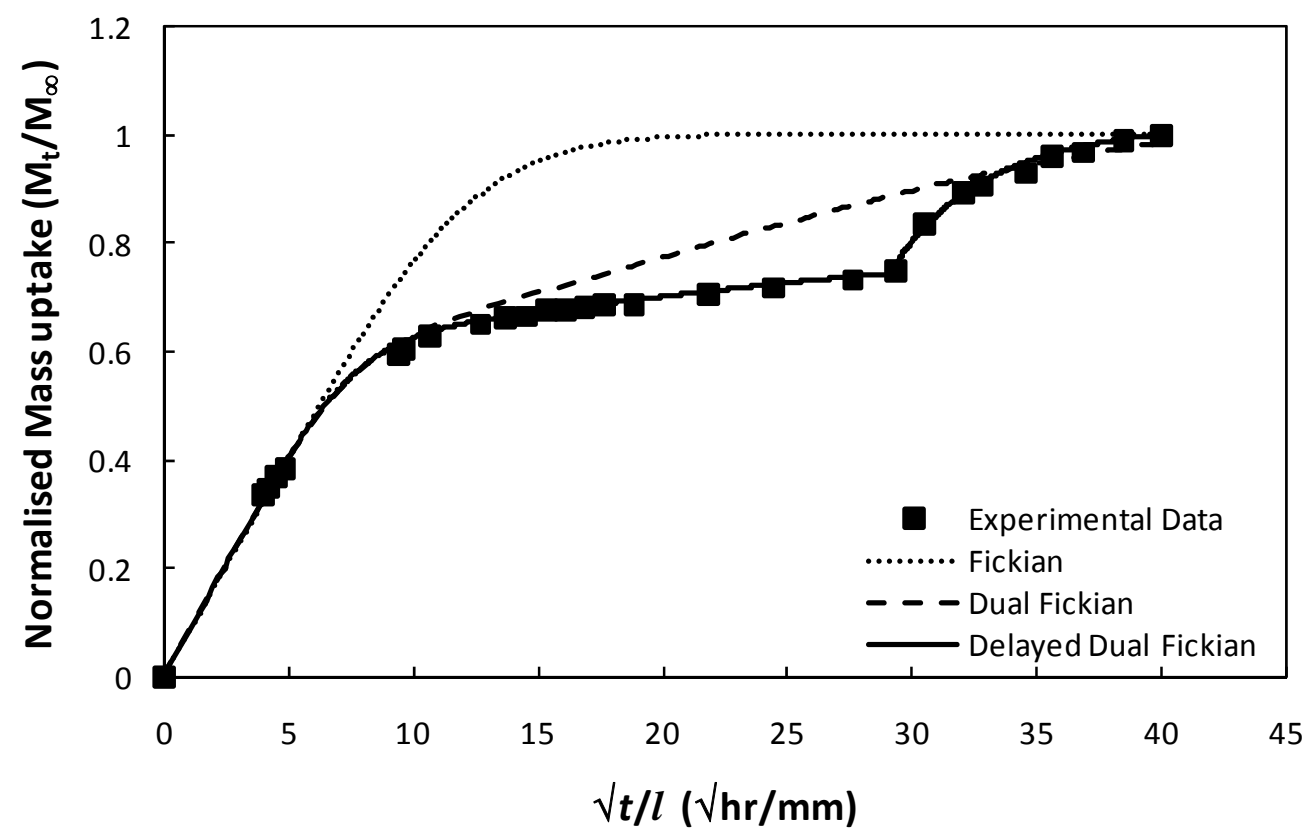

(a)

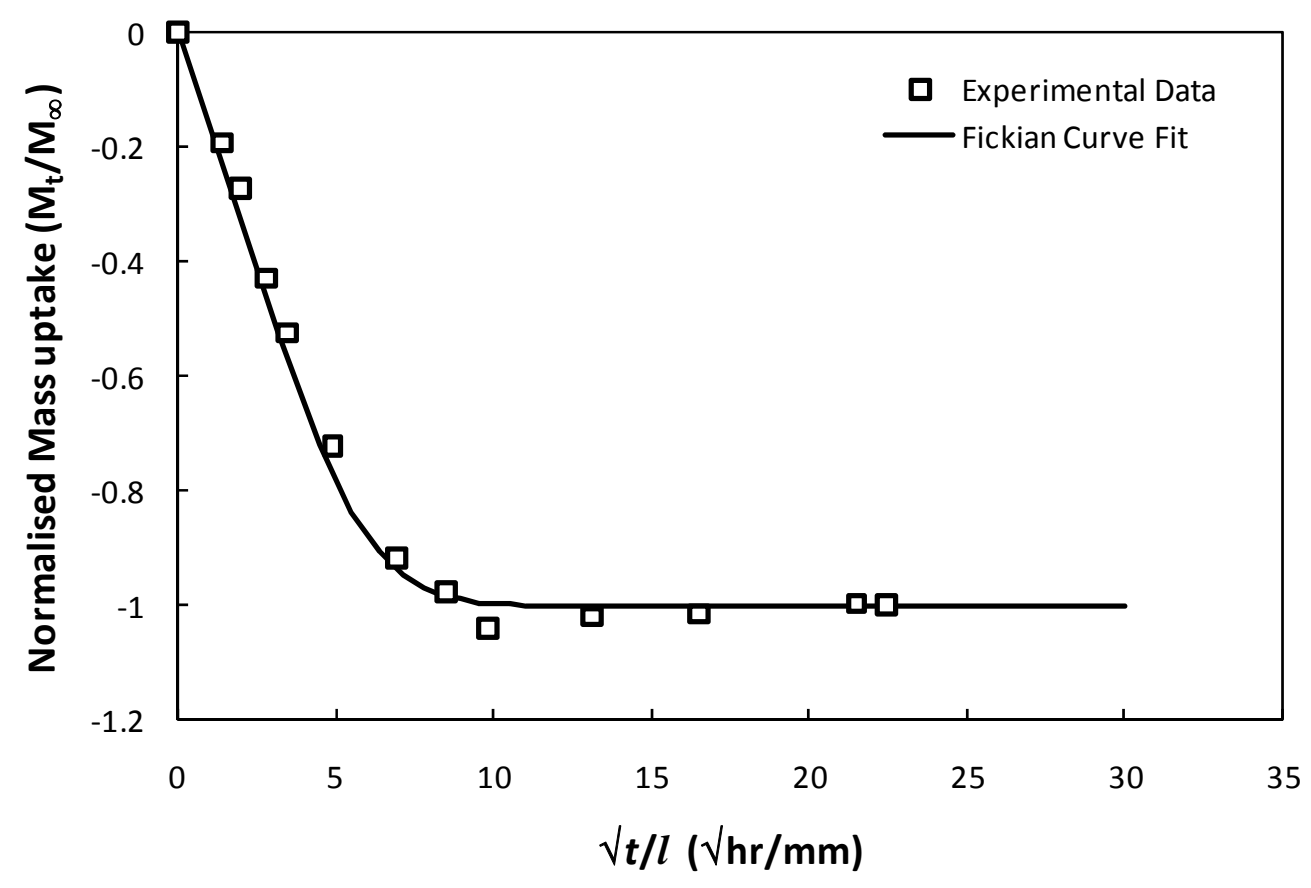

(b)

Figure 2: Curve fits of experimental moisture uptake for $1 \mathrm{~mm}$ thick samples when conditioned at $50^{\circ} \mathrm{C}$, immersed in water (a) absorption (b) desorption. 


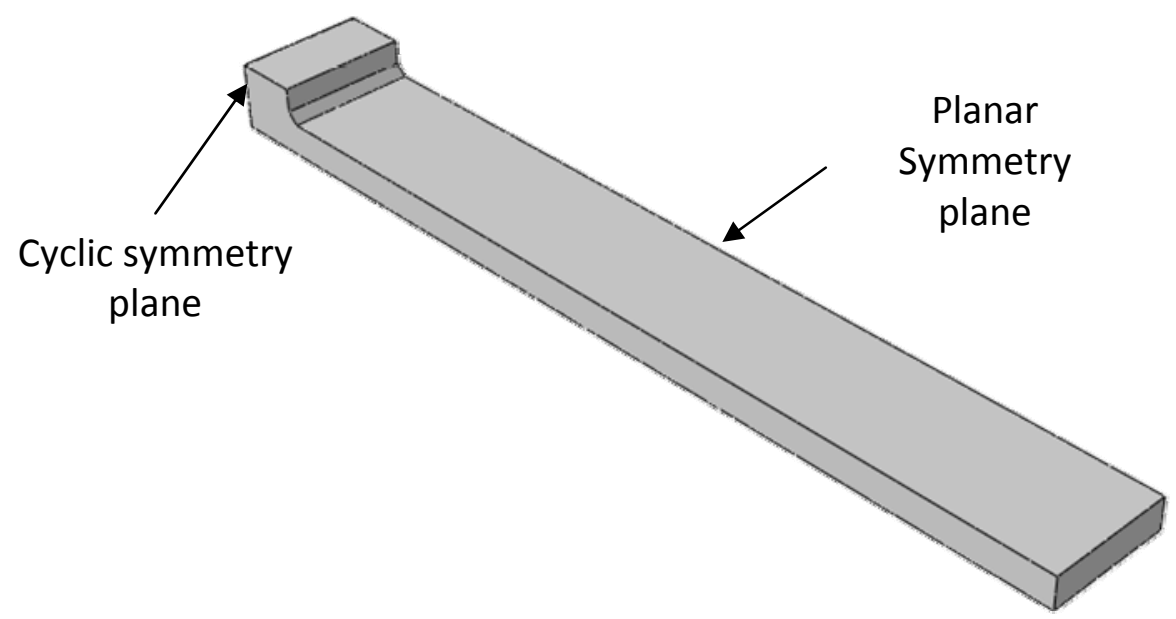

(a)

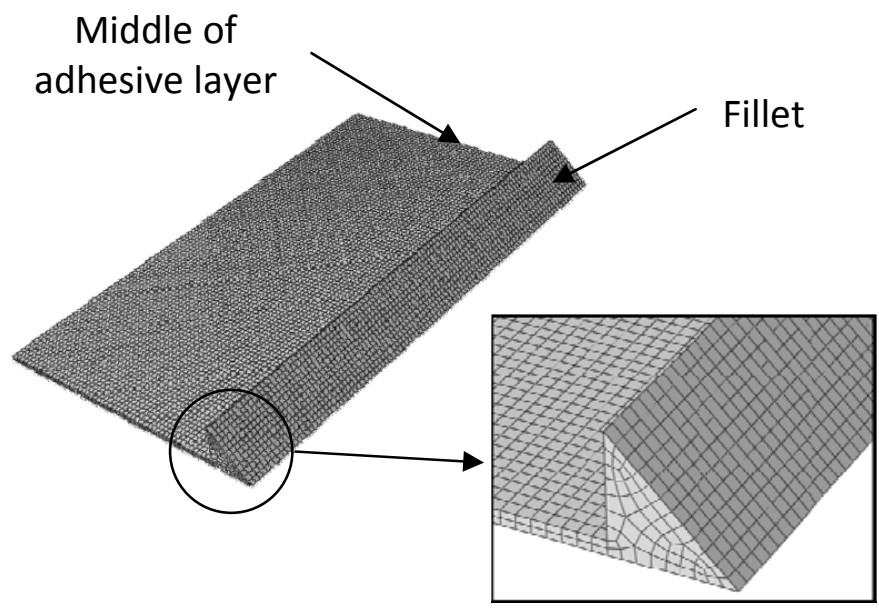

(b)

Figure 3: Finite element model of single lap joint (a) symmetry conditions applied to joint (b) detailed mesh of the adhesive layer 


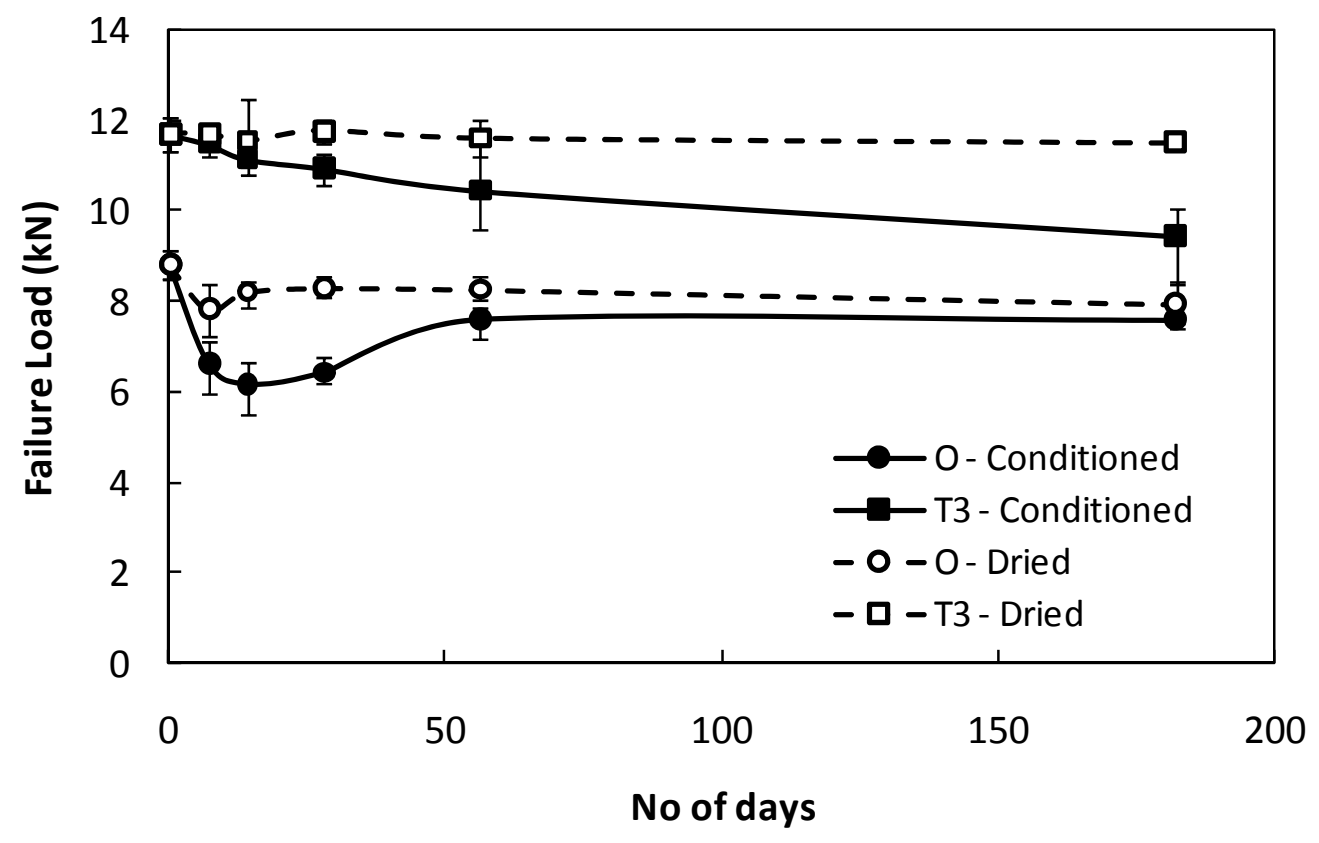

Figure 4: Failure load of single lap joints after conditioning at $50^{\circ} \mathrm{C}$, immersed in water.

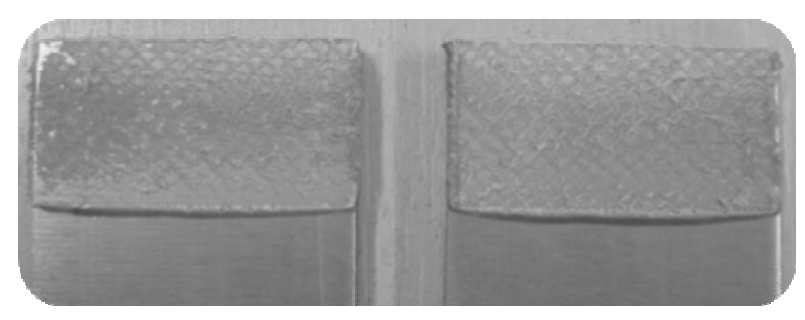

(a)

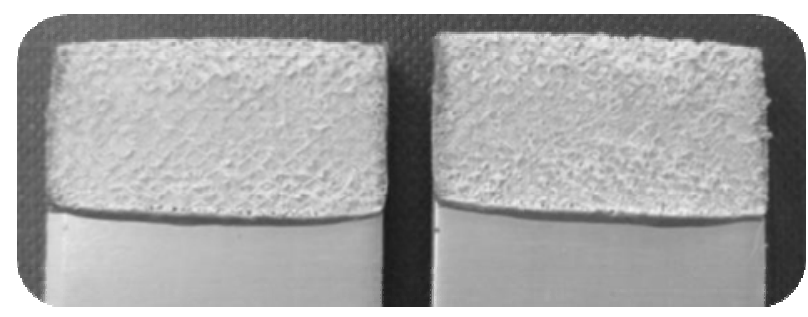

(b)

Figure 5: Cohesive failure in unconditioned single lap joints with adherends of type (a) Al2024 T3 (b) Al2024 O 


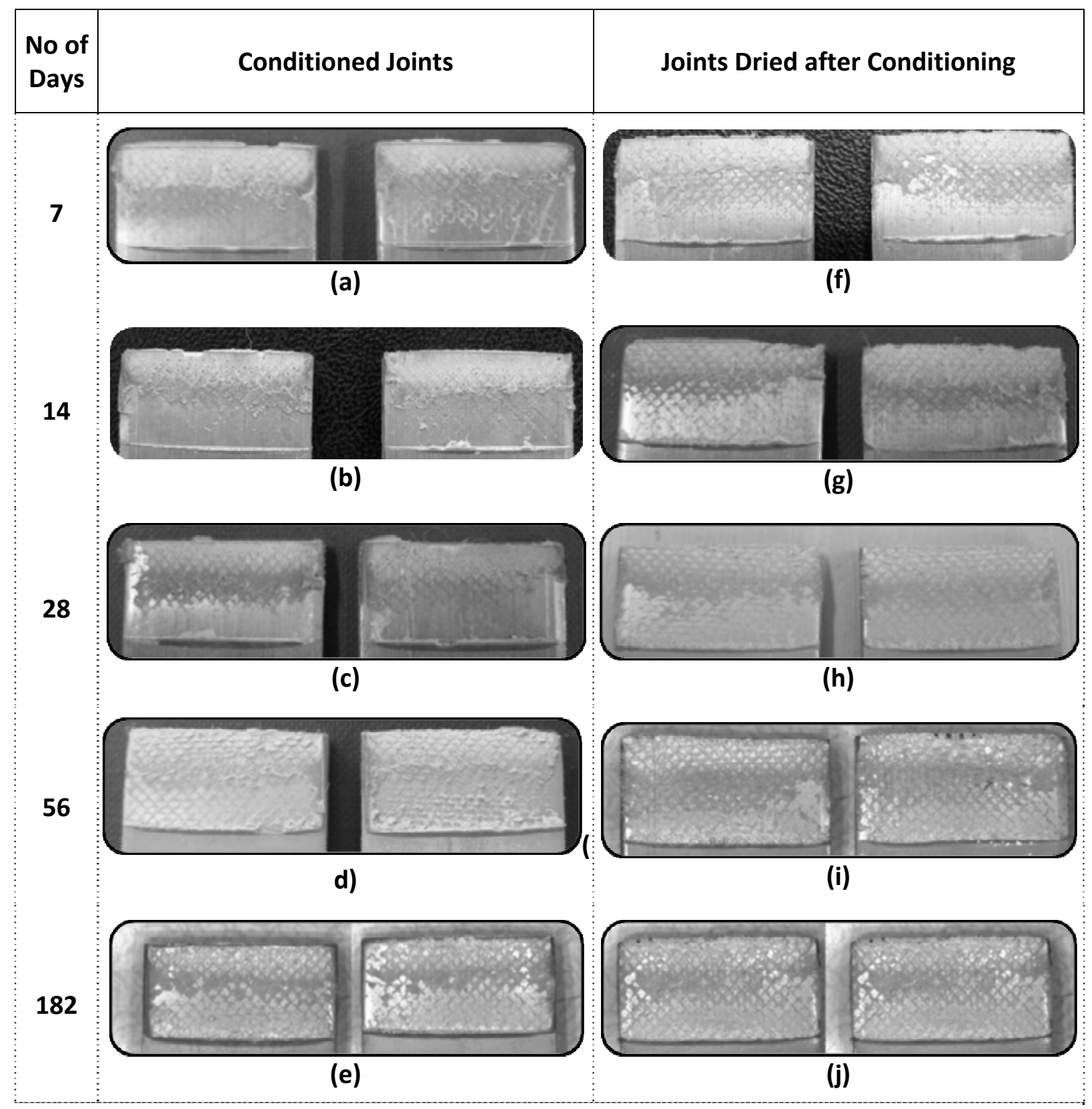

Figure 6: Failure surfaces of single lap joints, with Al2024 O adherends, after tensile testing. A set of joints was tested after conditioning in water at $50^{\circ} \mathrm{C}$, and other set was tested after drying. 


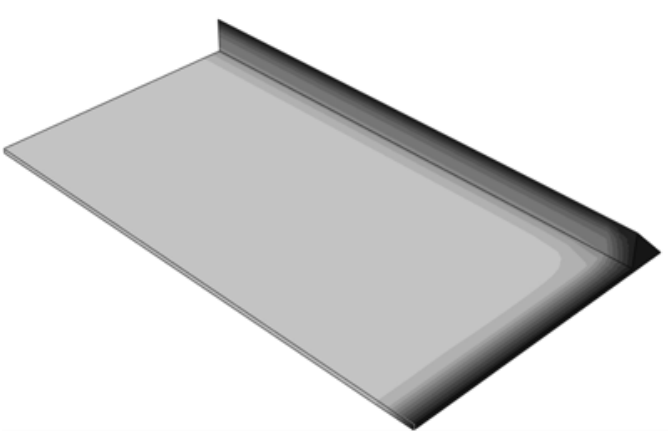

(a) 7 Days

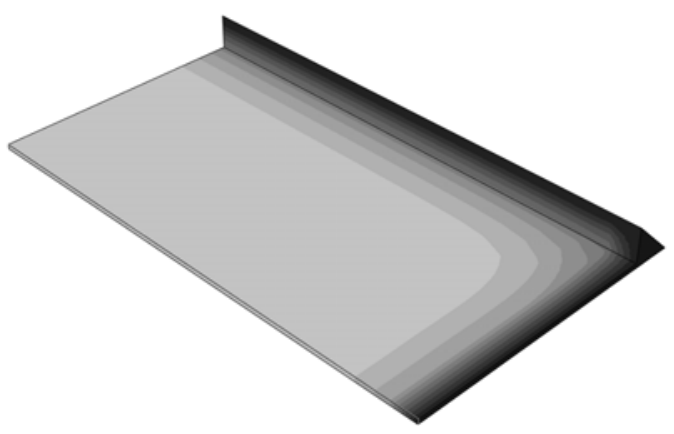

(c) 28 Days

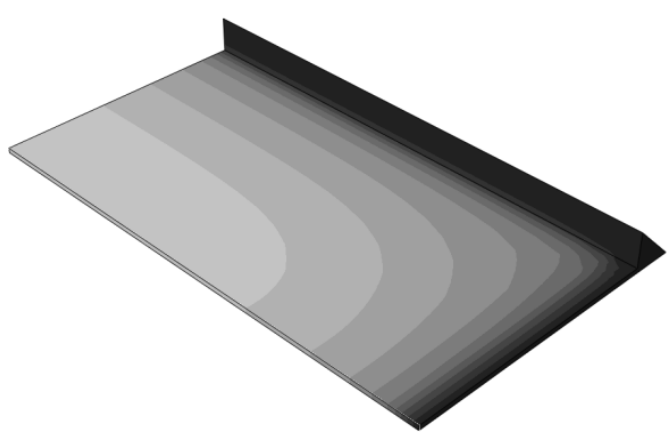

(e) 182 Days

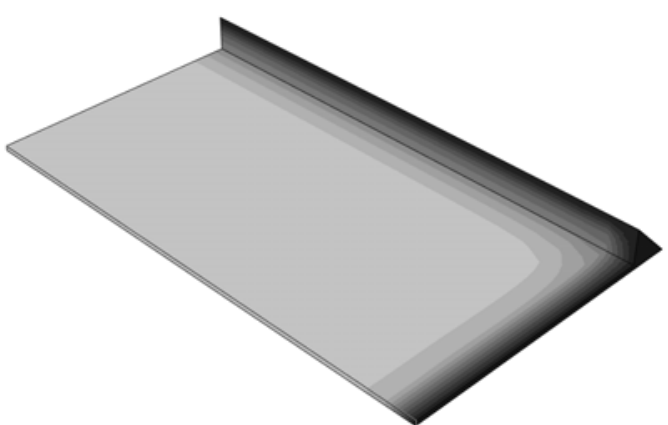

(b) 14 Days

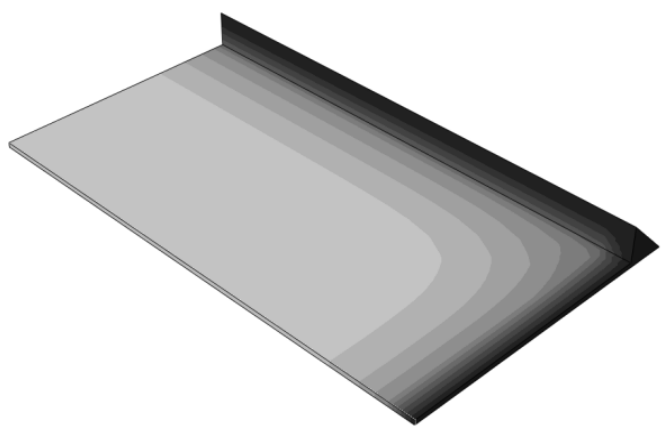

(d) 56 Days

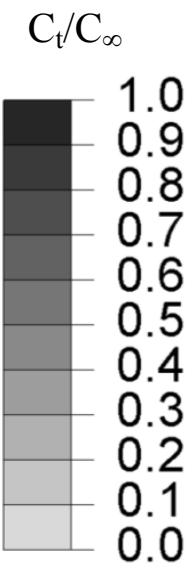

Figure 7: Normalised moisture concentration profiles in the adhesive layer after various intervals, when conditioned in water at $50^{\circ} \mathrm{C}$. 


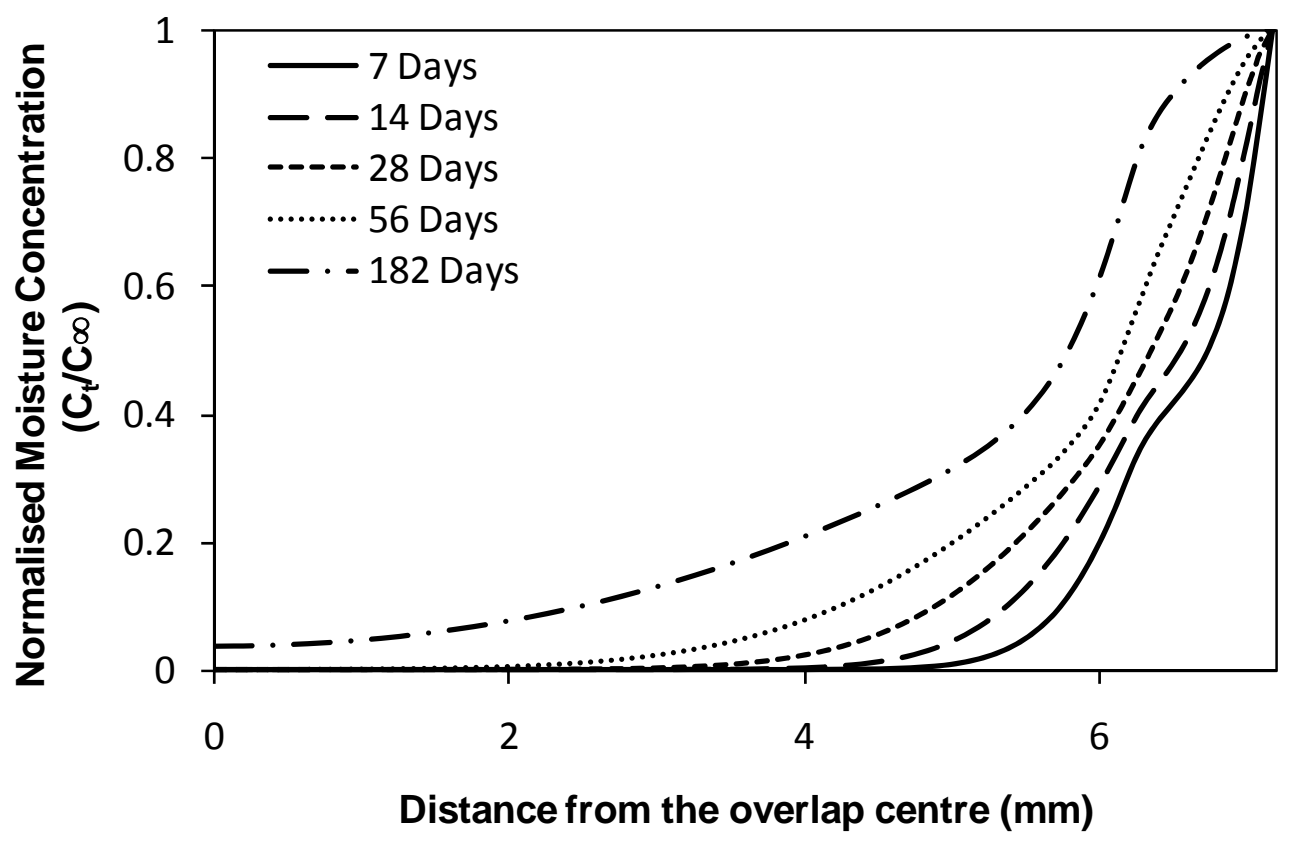

Figure 8: Normalised moisture concentration in the middle of the adhesive layer. 


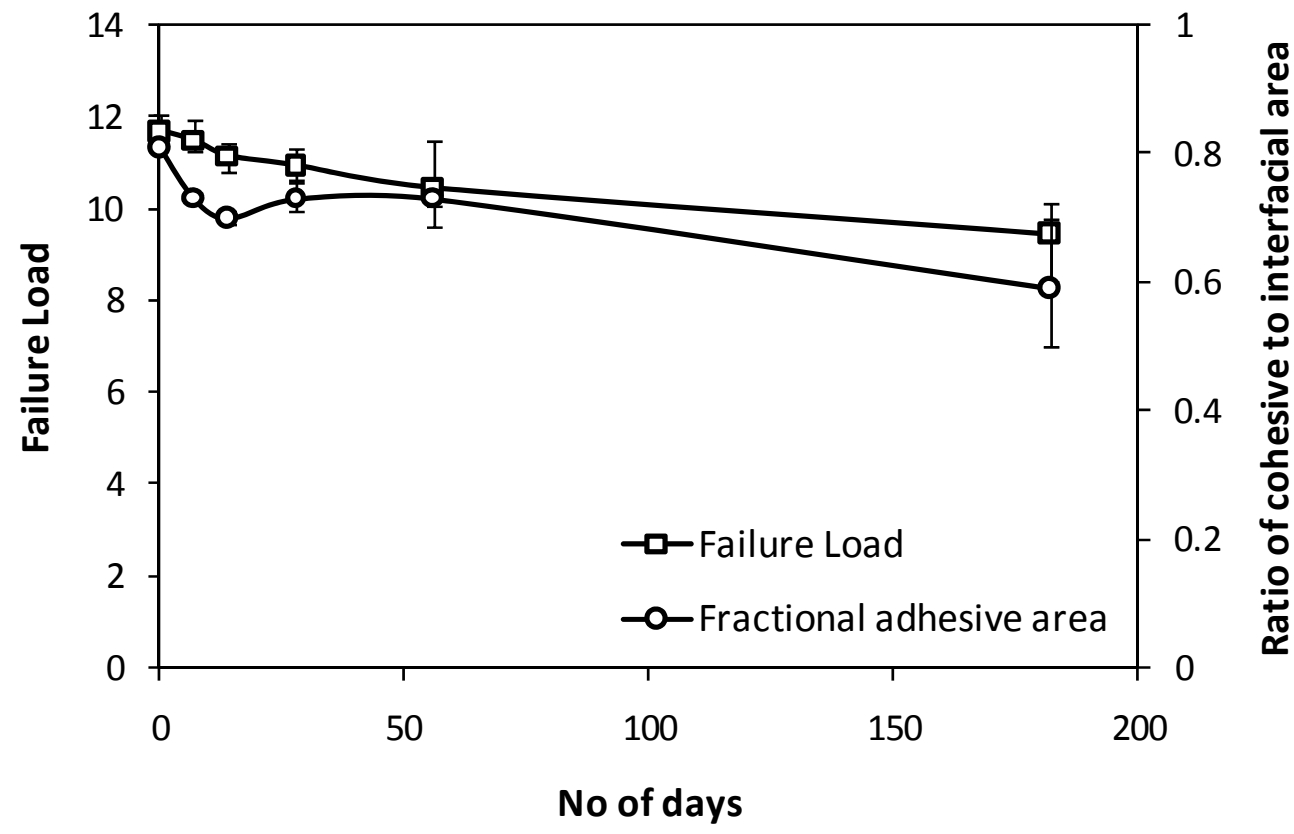

Figure 9: Comparison of failure load and failure type in Al2024 T3 joints after conditioning at $50^{\circ} \mathrm{C}$ in water.

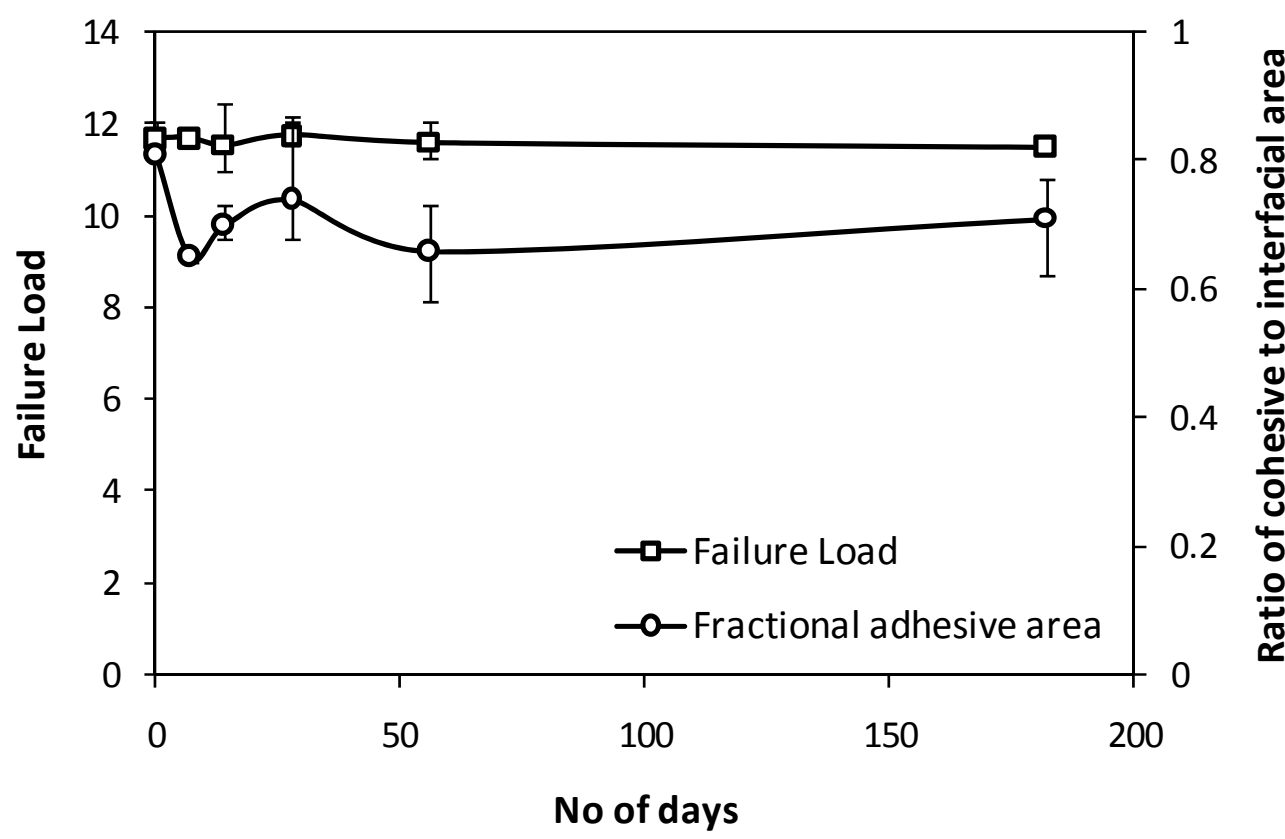

Figure 10: Comparison of failure load and failure type in Al2024 T3 joints after conditioning at $50^{\circ} \mathrm{C}$ in water and drying. 


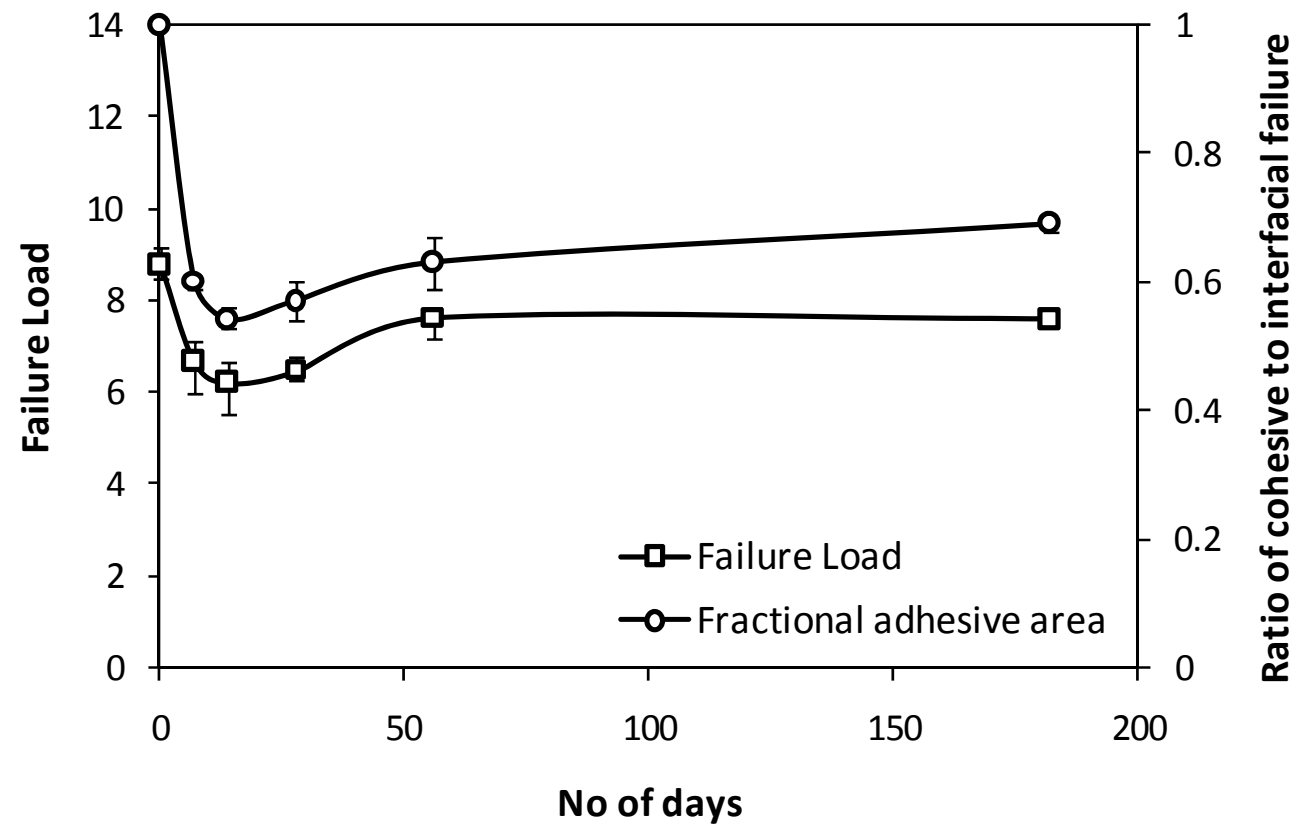

Figure 11: Comparison of failure load and failure type in Al2024 O joints after conditioning at $50^{\circ} \mathrm{C}$ in water.

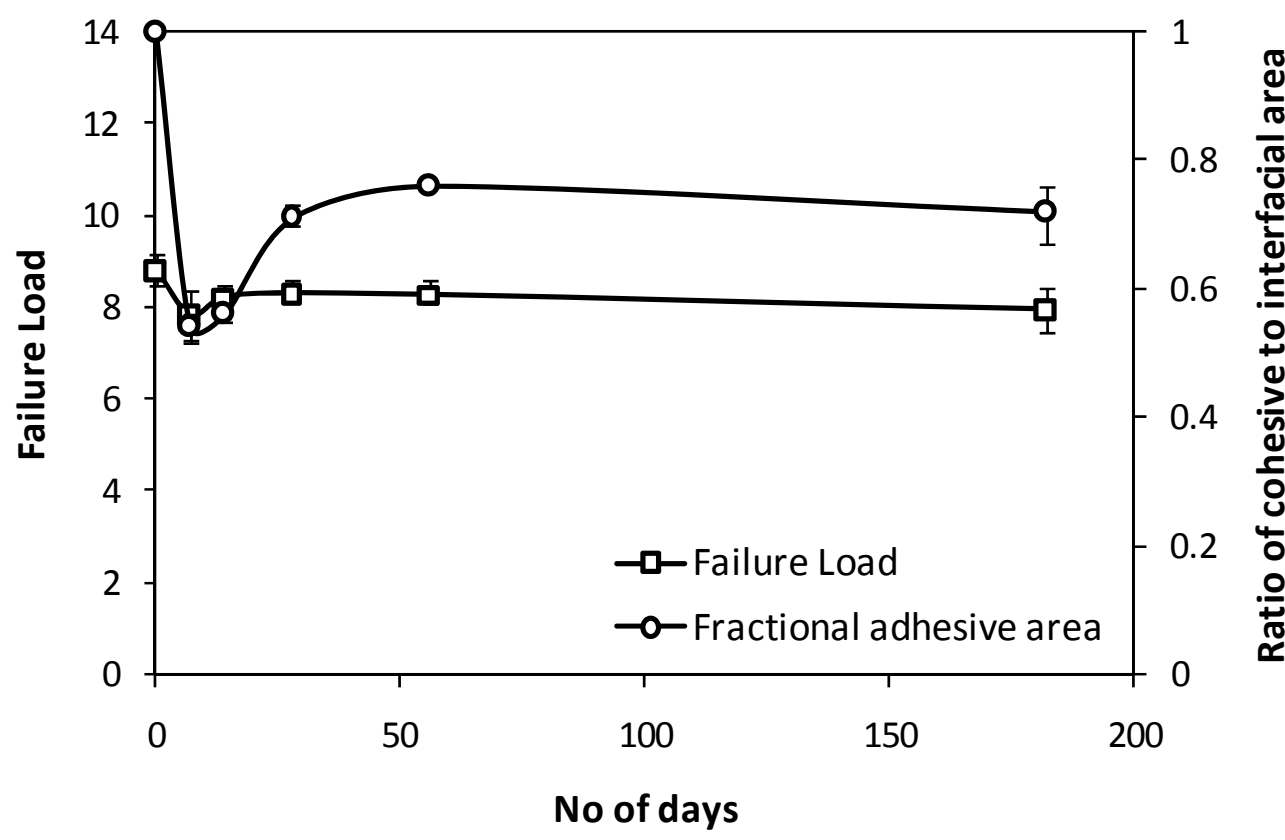

Figure 12: Comparison of failure load and failure type in Al2024 O joints after conditioning at $50^{\circ} \mathrm{C}$ in water and drying. 


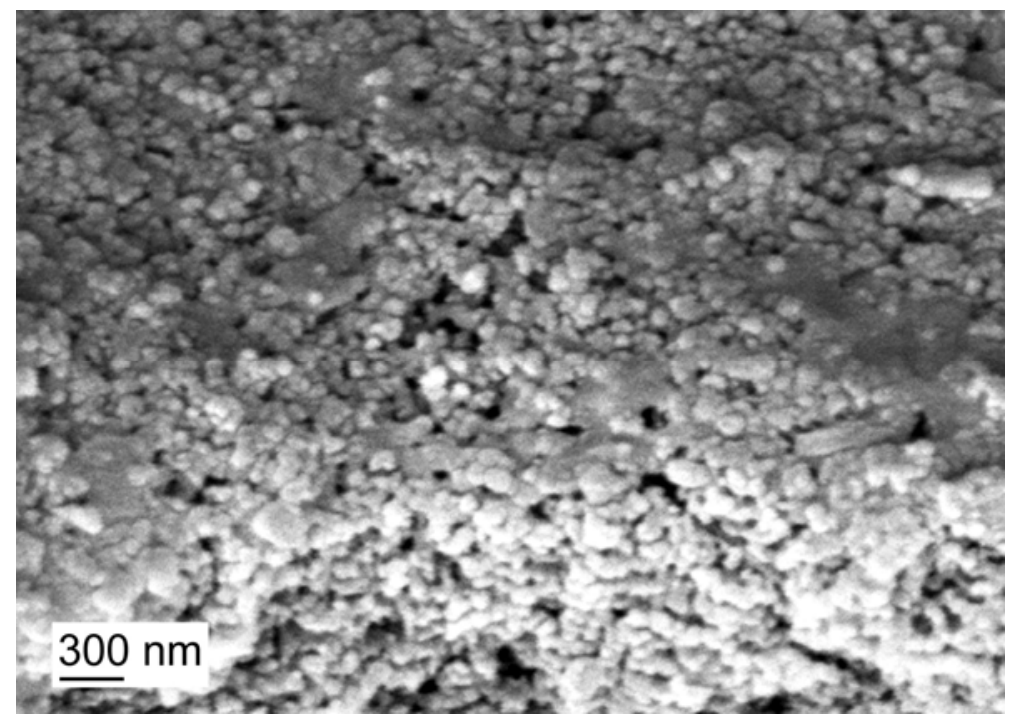

Figure 13: Scanning electron microscope image of porous oxide surface generated by ACDC pretreatment on aluminium 2024 T3.

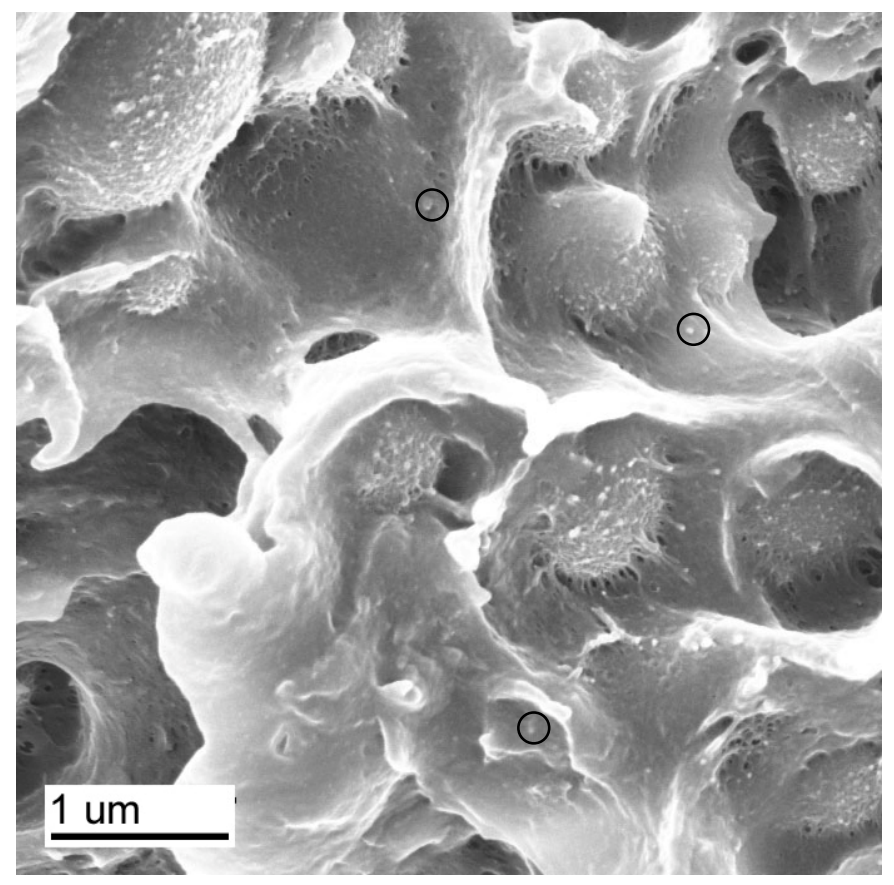

Figure 14: Failure surface of a joint made from primer BR127 only (no adhesive was used) when seen by SEM. The circles show the tiny strontium chromate particles. 


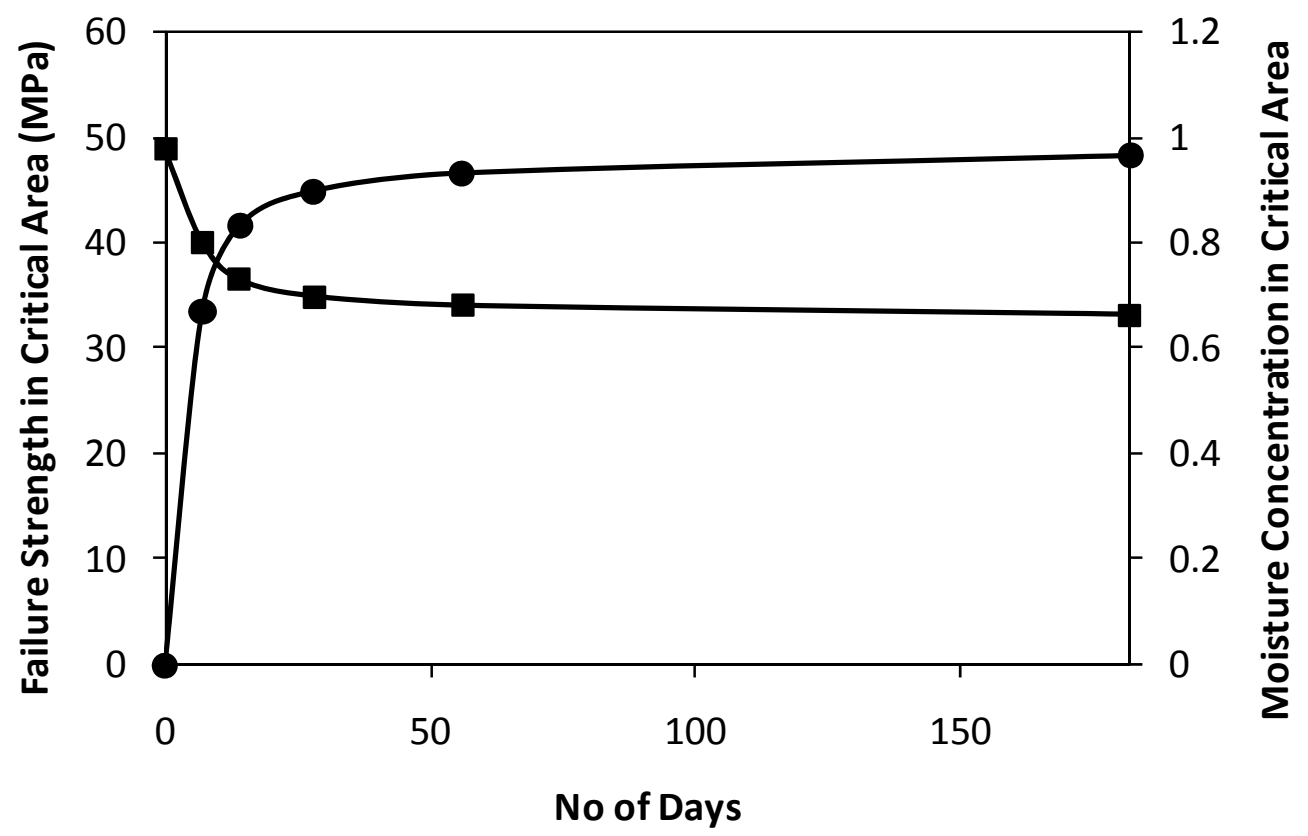

(a)

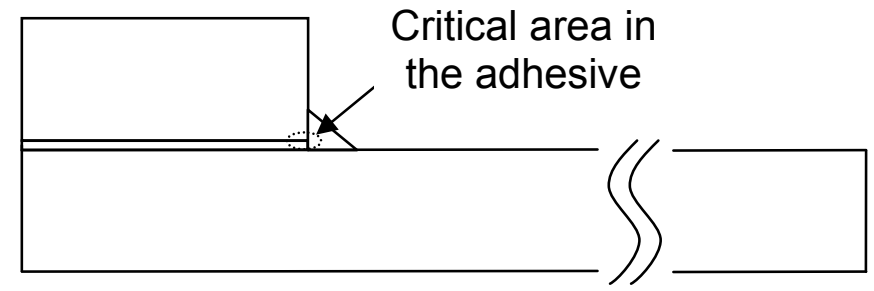

(b)

Figure 15: Failure strength and moisture concentration (a) change with conditioning time and (b) critical area in the adhesive (not to scale). 
Table 1: Coefficients of diffusion determined by curve fitting to experimental data.

Dual Fickian curve fit for absorption

\begin{tabular}{cccc}
\hline$D_{1}\left(\mathrm{~m}^{2} / \mathrm{s}\right)$ & $D_{2}\left(\mathrm{~m}^{2} / \mathrm{s}\right)$ & $M_{1 \infty}(\mathrm{wt} \%)$ & $M_{2 \infty}(\mathrm{wt} \%)$ \\
$9.75 \times 10^{-13}$ & $3.11 \times 10^{-14}$ & 1.78 & 1.92 \\
\hline
\end{tabular}

Delayed Dual Fickian curve fit for absorption

\begin{tabular}{cccccccc}
\hline$D_{1}$ & $D_{2}$ & $M_{1 \infty}(\mathrm{wt} \%)$ & $M_{2 \infty}$ & $t_{1}$ & $a$ & $b$ & $c$ \\
$\left(\mathrm{~m}^{2} / \mathrm{s}\right)$ & $\left(\mathrm{m}^{2} / \mathrm{s}\right)$ & & $(\mathrm{wt} \%)$ & $(\mathrm{sec})$ & & & \\
$\begin{array}{c}9.27 \times 10^{-} \\
13\end{array}$ & $4.75 \times 10^{-14}$ & 2.03 & 0.67 & $3.09 \times 10^{6}$ & $\begin{array}{c}-2.56 \times \\
10^{12}\end{array}$ & -4.26 & 0.85 \\
\hline
\end{tabular}

Fickian curve fit for desorption

\begin{tabular}{|c|c|}
\hline$D\left(\mathrm{~m}^{2} / \mathrm{s}\right)$ & $M_{\infty}(w t \%)$ \\
\hline $1.17 \times 10^{-12}$ & 3.7 \\
\hline
\end{tabular}


Table 2: Composition of failure surfaces when analysed with XPS.

\begin{tabular}{|c|c|c|c|c|c|c|}
\hline \multirow{2}{*}{ Joint Type } & \multirow{2}{*}{ Conditioning } & \multirow{2}{*}{$\begin{array}{l}\text { Failure } \\
\text { Surface }\end{array}$} & \multicolumn{4}{|c|}{ Failure Surface Composition (Atomic \%) } \\
\hline & & & $\mathrm{Al}$ & $\mathrm{O}$ & $\mathrm{C}$ & $\mathrm{N}$ \\
\hline $\begin{array}{l}\text { Anodised } \\
\text { Substrate }\end{array}$ & & & 27.2 & 48.4 & 20.8 & - \\
\hline \multirow[t]{4}{*}{ Al2024 T3 } & Unconditioned & Metal & 1.2 & 15.1 & 81.0 & 1.3 \\
\hline & & Adhesive & 0.0 & 16.3 & 82.5 & Trace \\
\hline & 56 days in water & Metal & 1.9 & 14.6 & 82.4 & 1.1 \\
\hline & & Adhesive & 1.4 & 13.3 & 84.2 & 1.2 \\
\hline \multirow[t]{6}{*}{ Al2024 O } & Unconditioned & Metal & 0.5 & 14.2 & 83.6 & 0.8 \\
\hline & & Adhesive & 0.0 & 13.6 & 85.8 & Trace \\
\hline & 14 days in water & Metal & 13.9 & 27.6 & 56.1 & 0.4 \\
\hline & & Adhesive & 2.6 & 17.3 & 80.0 & 1.0 \\
\hline & $\begin{array}{l}\text { Dried after } 7 \text { days } \\
\text { in water }\end{array}$ & Metal & 18.0 & 27.8 & 49.9 & 0.0 \\
\hline & & Adhesive & 0.0 & 16.8 & 82.9 & Trace \\
\hline
\end{tabular}

